\title{
Detection of a Quantitative Trait Locus for Intramuscular Fat Accumulation Using the OLETF Rat
}

\author{
Hideki TANOMURA ${ }^{1)}$, Takeshi MIYAKE ${ }^{1)}$, Yukio TANIGUCHI ${ }^{1)}$, Noboru MANABE ${ }^{2)}$, Hiroyuki KOSE ${ }^{3)}$, \\ Kozo MATSUMOTO ${ }^{3)}$, Takahisa YAMADA ${ }^{1)}$ and Yoshiyuki SASAKI ${ }^{1) *}$ \\ ${ }^{1)}$ Laboratories of Animal Breeding and Genetics and ${ }^{2)}$ Anatomy and Cell Biology, Graduate School of Agriculture, Kyoto University, \\ Sakyoku, Kyoto 606-8502 and ${ }^{3)}$ Institute for Animal Experimentation, University of Tokushima School of Medicine, Kuramoto, Tokushima \\ 770-8503, Japan
}

(Received 28 June 2001/Accepted 13 September 2001)

ABSTRACT. The Otsuka Long-Evans Tokushima Fatty (OLETF) rat is an animal model for obese type 2 diabetes. We showed that the OLETF rat exhibits higher levels of intramuscular fat content in Musculus longissimus as compared to the Fischer-344 (F344) rat. Our investigation was designed to identify quantitative trait loci (QTLs) contributing to the increased levels of intramuscular fat content by performing a whole-genome search using $108 \mathrm{~F}_{2}$ intercross obtained by mating the OLETF and the F344 rats. We identified one QTL responsible for intramuscular fat accumulation on rat chromosome 1 with a maximum lod score of 3.4, which accounts for $5 \%$ of the total variance. As expected, the OLETF allele corresponds to the increased levels of intramuscular fat content. KEY WORDS: animal model, intramuscular fat, obesity, OLETF rat, QTL.

J. Vet. Med. Sci. 64(1): 45-50, 2002

Intramuscular fat, so called marbling is an important factor for evaluation of beef quality, and intramuscular fat accumulation is the main target in beef industries, particularly in Japan. Marbling is more markedly observed in the Japanese Black cattle than other breeds of cattle [2,34] and heritability for marbling is very high [18], indicating an important genetic component in intramuscular fat accumulation. Recently, Miyake et al. [16] have reported presence of a major gene for intramuscular fat accumulation in cattle. However, there is still no more than one report on the mapping of quantitative trait loci (QTLs) representing major genes for intramuscular fat accumulation in cattle [3]. Bovine studies for such QTLs are complicated by genetic heterogeneity, environmental influences, and difficulty in constructing experimental crosses. The use of inbred animal models of intramuscular fat accumulation is an essential component for investigation of such QTLs using the strategy of molecular and statistical genetics.

The Otsuka Long-Evans Tokushima Fatty (OLETF) rat was established by selective breeding based on impaired glucose tolerance [11]. The OLETF rat develops obese type 2 diabetes [11]. Several QTL analyses on obesity and type 2 diabetes in this rat have been reported $[9,17,20,25,29$, 30]. Among these analysis, there is our previous study that have identified six QTLs responsible for adiposity indices of fat pads on rat chromosomes 2 (Obs 1 for mesenteric fat), 4 (Obs2 for retroperitoneal fat), 8 (Obs3 for mesenteric fat), 9 (Obs4 for retroperitoneal fat), and 14 (Obs5 and Obs6 for retroperitoneal fat) [20]. This study demonstrated that the adiposity indices of individual fat pads were under the control of different genes.

\footnotetext{
* Correspondence to: Sasaki, Y., Laboratory of Animal Breeding and Genetics, Graduate School of Agriculture, Kyoto University, Sakyoku, Kyoto 606-8502, Japan.
}

In the present study, we have demonstrated that the OLETF rat possesses higher levels of intramuscular fat content in Musculus longissimus than the Fischer-344 (F344) rat. Thus, we set out to search for evidence of QTLs affecting intramuscular fat accumulation by using the interval mapping method in $108 \mathrm{~F}_{2}$ progenies obtained by mating the OLETF and the F344 rats. We have identified one QTL responsible for intramuscular fat accumulation on chromosome 1 .

\section{MATERIALS AND METHODS}

Genetic crosses: All of the $\mathrm{F}_{1}$ rats were generated by female OLETF rats bred with male $\mathrm{F} 344$ rats, and $\mathrm{F}_{2}$ progeny were obtained by intercrossing the $F_{1}$ rats. Male rats were used for genetic studies. The difference in degree of adiposity between OLETF and F344 rat strains is more markedly observed in males than in females $[11,20]$. Thus, using males was presumed to exclusively maximize the quantitative difference of intramuscular fat content between the two strains for greater statistical power to detect QTLs [12]. All rats were kept under specific pathogen-free conditions. The temperature $\left(21 \pm 2{ }^{\circ} \mathrm{C}\right)$, humidity $(55 \pm 5 \%)$, and air conditioning were all controlled. Rats had free access to tap water and standard laboratory chow (CRF-1; Oriental Yeast, Tokyo, Japan) and were maintained on a 12-hr light and dark cycle (6 AM/6 PM).

Phenotyping: Rats were killed under deep anesthesia at 35 weeks of age. Immediately after exsanguination, the first to third lumbar region of Musculus longissimus was removed from the right side of the carcass and rapidly frozen in dry ice-cooled isopentane. Five serial coronal sections ( $6 \mu \mathrm{m}$ thick) were obtained at intervals of $180 \mu \mathrm{m}$ from frozen Musculus longissimus, and the sections stained with Oil Red $\mathrm{O}$ for the presence of lipid droplets in adipocyte 
cytoplasm. The section area, occupied by cells positive for Oil Red $\mathrm{O}$ staining, were measured in a given area $\left(1 \mathrm{~cm}^{2}\right)$ using MacSCOPE computer program. The fat staining-positive area expressed as an absolute value or as a percentage of the body weight was used as the quantitative phenotype of the levels of intramuscular fat content. The body weight was measured before the sacrifice of rats. The tail was cut and frozen at $-80^{\circ} \mathrm{C}$ for subsequent isolation of high-molecular-weight DNA. This study conformed to the guidelines for the care and use of laboratory animals of Kyoto University.

Genotyping: DNA was isolated from the tail using phenol-chloroform extraction. The $($ OLETF female $\times$ F344 male) $F_{2}$ progeny were genotyped by polymerase chain reaction (PCR) amplification of microsatellites with the primers purchased from Research Genetics, Inc. (Huntsville, AL), according to a previous report [17].

Data analysis: Genetic markers were mapped relative to one another with the MAPMAKER/EXP computer package [14]. A candidate for laboratory typing error was identified by an error detection procedure on the MAPMAKER/EXP computer package, which is a systematic method that incorporates the possibility of error into the usual likelihood model for linkage analysis [15]. Potential errors were rechecked against photographs, and, where necessary, the genotyping was repeated. QTLs affecting phenotypes were mapped relative to genetic markers with the MAPMAKER/ QTL computer package [22]. We selected a lod score of 2.8 as the genome-wide threshold for establishment of linkage [13]. Phenotypic comparisons for different genotypic groups were performed by using a Student's $t$ test or an analysis of variance (ANOVA) with a post hoc test using
Scheffe's $F$ test (StatView, SAS Institute, Inc., Cary, NC). Multiple QTL analysis was conducted with the 'simultaneous search' function of MAPMAKER/QTL computer package. Briefly, identified QTL is 'fixed', removing that portion of the variance that is explained by that locus from the subsequent analysis. The genome is then rescanned to identify additional QTLs. Multiple QTL analysis was also performed by MQM-mapping on the MapQTL computer program [6, 7]. QTL-likelihood plots were produced by using the marker that flanks closest to the lod score peak of identified QTL as cofactor for QTL mapping on the MapQTL computer program. Identified QTL was confirmed by QTL mapping in which marker closest to the lod score peak of the identified QTL was used as cofactor except for the marker flanking the interval under study. Although, in case of a few cofactors in multiple-QTL models, there may be no clear difference in the mapping efficacy between multiple QTL analyses with MAPMAKER/QTL and with MapQTL, the two analyses were carried out to confirm additional QTLs.

Examination of syntenic conservation among rat, mouse, and human: Syntenic conservation between rat, mouse, and human chromosomes were investigated on the basis of the radiation hybrid map of rat [28], the rat linkage map [1, 5, 31], the human obesity gene map [23], and several Web site databases (http://ratmap.gen.gu.se/; http://wwwgenome.wi.mit.edu:80/ftp/distribution/; and http:// www.otsuka.genome.ad.jp/ratmap).

\section{RESULTS}

We stained the coronal sections of Musculus longissimus

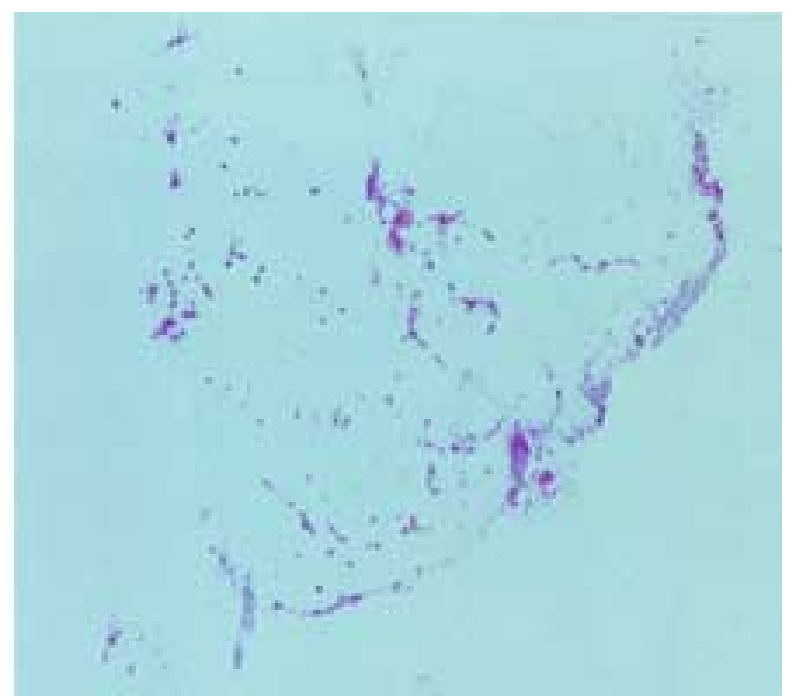

OLETF

Fig. 1. Oil Red O staining of the coronal sections of Musculus longissimus from OLETF and F344 rats. The area positive for Oil Red O staining is shown by red color. 
Table 1. Phenotypic characteristics of parental strains, $F_{1}$ progeny, and $F_{2}$ progeny

\begin{tabular}{ccccc}
\hline Phenotype & OLETF & F344 & $\mathrm{F}_{1}$ progeny & $\mathrm{F}_{2}$ progeny \\
\hline Number of animals & 7 & 7 & 7 & 108 \\
Fat staining-positive area $\left(\mathrm{mm}^{2}\right)$ & $3.26 \pm 0.52^{\mathrm{a})}$ & $1.34 \pm 0.38^{\mathrm{b})}$ & $2.44 \pm 1.03^{\mathrm{c})}$ & $2.52 \pm 1.55$ \\
$\begin{array}{c}\text { Fat staining-positive area/body } \\
\text { weight }\left(\mathrm{mm}^{2} / \mathrm{g} \times 10^{-3}\right)\end{array}$ & $5.01 \pm 2.29^{\mathrm{a})}$ & $3.62 \pm 2.19^{\mathrm{b})}$ & $4.06 \pm 1.71^{\mathrm{c})}$ & $4.74 \pm 2.99$ \\
Body weight $(\mathrm{g})$ & $650.2 \pm 31.2^{\mathrm{a})}$ & $375.6 \pm 20.0^{\mathrm{b})}$ & $600.2 \pm 24.3^{\mathrm{c})}$ & $531.1 \pm 48.4$
\end{tabular}

Phenotypes are given as mean \pm SD. Only male rats were phenotyped at 35 weeks of age. Phenotypes in the parental strains and $\mathrm{F}_{1}$ progeny were compared by a one-way analysis of variance with Scheffe's $F$ analysis as post hoc test. Means labeled with different superscripts are significantly different from one another $(P<0.05)$.

from OLETF and F344 rats with Oil Red O. The section area, occupied by cells positive for Oil Red O staining, was visually larger in OLETF than in F344 (Fig. 1), suggesting an increase of intramuscular fat content in Musculus longissimus of the OLETF rat.

We crossed female OLETF rats with male F344 rats to generate $F_{1}$ progeny which, in turn, were used to generate 108 male $F_{2}$ progeny. Only males, 35 weeks of age, from the parental strains, the $7 \mathrm{~F}_{1}$ progeny, and the $108 \mathrm{~F}_{2}$ progeny were phenotyped for the levels of intramuscular fat content, that is the fat staining-positive area expressed as an absolute value or as a percentage of the body weight (Table 1). The fat staining-positive area as an absolute value was increased in the OLETF rat as compared with the F344 rat, and the OLETF and F344 rats showed clear statistical difference in this phenotype $(P<0.0001)$. The $\mathrm{F}_{1}$ progeny showed that the trait of this increased positive area is inherited in an additive manner. Compared with the $F_{1}$ progeny, the $F_{2}$ progeny showed similar means but larger variance in the phenotype. The broad, continuous distributions without evidence of distinct groups in this phenotype among the $\mathrm{F}_{2}$ progeny suggest that this trait is polygenically regulated. The distribution of the fat staining-positive area as a percentage of the body weight showed a similar trend to that expressed as an absolute value in the four genetic groups of the parental strains and the $F_{1}$ and $F_{2}$ progenies. These indicate the existence of increased levels of intramuscular fat content in the OLETF rat.

To identify the QTL(s) affecting the increased levels of intramuscular fat content, we carried out a total genome scan on the $\mathrm{F}_{2}$ progeny, using a set of 222 informative simple sequence length polymorphisms between the OLETF and the F344 rats. The markers were on average $7.6 \mathrm{cM}$ apart, and approximately $94 \%$ of the genome (based on a genetic length of $1705 \mathrm{cM}$ calculated in this study) was within $10 \mathrm{cM}$ of an informative marker. No discrepancies were found between maps constructed in this study and the maps reported in Rat Genome Database (http:// rgd.mcw.edu/). Phenotypic and genotypic data were analyzed for linkage with MAPMAKER/QTL [22]. According to the criteria set by Lander and Kruglyak [13], we found statistical evidence for one QTL affecting the increased levels of intramuscular fat content on chromosome 1. This locus, between D1Mit7 and D1Rat123 showed a maximum lod score of 3.4 for the fat staining-positive area and a maximum lod score of 3.2 for the fat staining-positive area/body weight, accounting for $5 \%$ of the fat staining-positive area variance and $7 \%$ of the fat staining-positive area/body weight variance in our cross (Fig. 2). The inheritance pattern at the locus was consistent with the OLETF alleles acting in a recessive mode of inheritance to increase the levels of intramuscular fat content (Table 2). Multiple QTL analysis with MAPMAKER/QTL indicated that no other QTLs were detected that statistically increased the baseline score of the chromosome 1 QTL, nor was any additional variance explained after fixing the chromosome 1 QTL in the $108 \mathrm{~F}_{2}$ progeny. Additionally, application of MQM-mapping, which is a mapping method based on multiple-QTL models $[6,7]$, has not led to a revelation of additional QTLs other than the chromosome 1 QTL. Note that no statistical evidence for an X-linked locus affecting the increased levels of intramuscular fat content was found by an analysis of variance.

\section{DISCUSSION}

In the present study, we demonstrated that the OLETF rat exhibits intramuscular fat accumulation and identified one QTL influencing this trait on chromosome 1 using the $F_{2}$ progeny obtained from the cross of the OLETF and the F344 rats. Our previous study reported QTL analysis on mesenteric, retroperitoneal, and epididymal fat accumulation in the OLETF rat [20]. The maximum lod scores for the adiposity indices of the mesenteric, retroperitoneal, and epididymal fat pads, respectively, in genomic region of the chromosome 1 QTL detected for intramuscular fat accumulation in this study, were $0.01,2.01$, and 0.03 , suggesting that this QTL has no impact on mesenteric, retroperitoneal, and epididymal fat accumulation in the OLETF rat. Therefore, the chromosome 1 QTL seems to exert an effect on fat storage only in intramuscular fat tissue. This has been noted by our previous report on obesity loci, that identified two QTLs, Obs1 (chromosome 2) and Obs3 (chromosome 8), and four QTLs, Obs2 (chromosome 4), Obs4 (chromosome 9), Obs5 (chromosome 14), and Obs6 (chromosome 14), as affecting mesenteric and retroperitoneal fat accumulation in the OLETF rat, respectively [20]. Analyses of obesity QTLs in polygenic mouse models have also demonstrated 


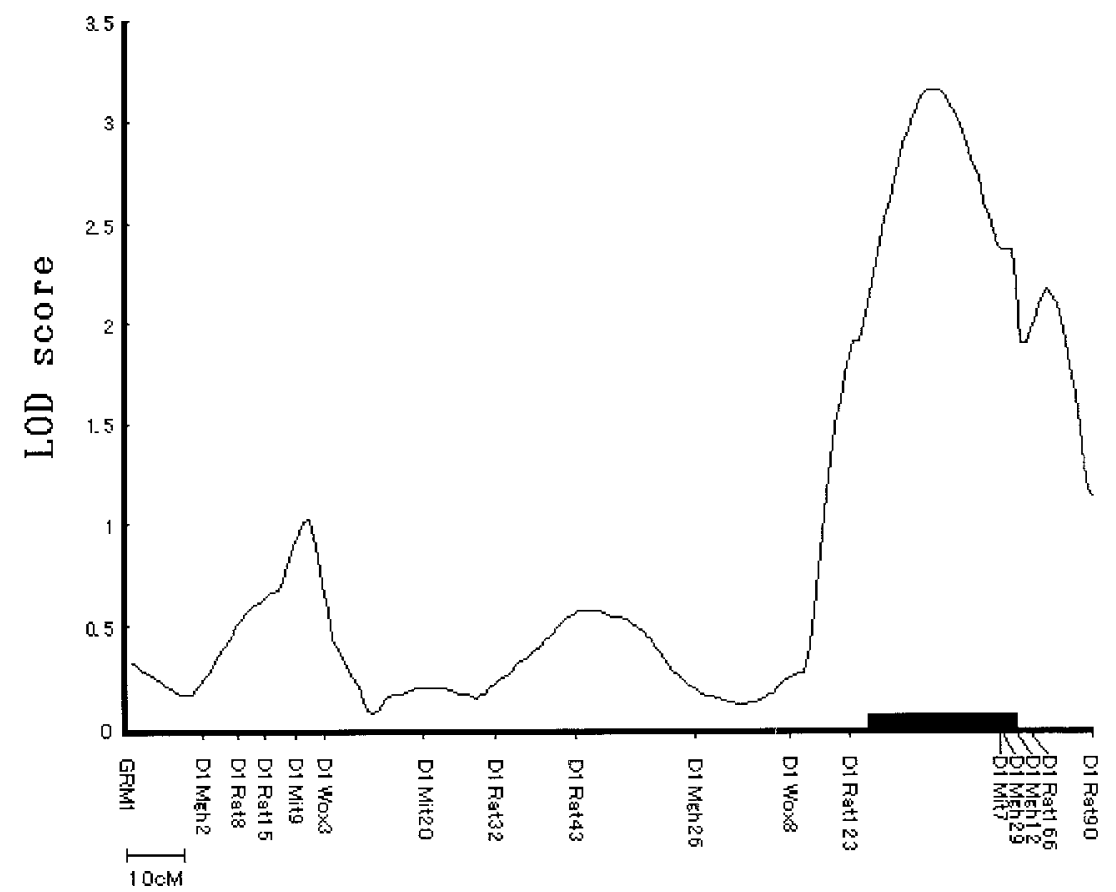

Fig. 2. Scan for lod score for linkage to fat staining-positive area expressed as an absolute value on chromosome 1 . The abscissa labels indicate microsatellite markers used for the linkage analysis, and map distances in centimorgans determined by the Kosambi map function are represented. The 1-lod support interval is shown as black bar. The lod scores were calculated by the MAPMAKER/QTL program at 2-cM intervals spanning the distance between each pair of polymorphic markers.

Table 2. Comparison of means of fat staining-positive area and fat staining-positive area/body weight in $\mathrm{F}_{2}$ progeny based on genotype at the chromosome 1 QTL

\begin{tabular}{cccc}
\hline Phenotype & \multicolumn{3}{c}{ Genotype } \\
\cline { 2 - 4 } & OLETF/OLETF & OLETF/F344 & F344/F344 \\
\hline Number of animals & 20 & 54 & 21 \\
Fat staining-positive area $\left(\mathrm{mm}^{2}\right)$ & $3.33 \pm 2.12^{\mathrm{a})}$ & $2.26 \pm 1.16^{\mathrm{b})}$ & $2.50 \pm 1.61^{\mathrm{b})}$ \\
Fat staining-positive area/body & $6.20 \pm 4.05^{\mathrm{a})}$ & $4.29 \pm 2.12^{\mathrm{b})}$ & $4.89 \pm 3.31^{\mathrm{b})}$ \\
weight $\left(\mathrm{mm}^{2} / \mathrm{g} \times 10^{-3}\right)$ & $543.0 \pm 46.1)^{\mathrm{a}}$ & $527.9 \pm 49.9^{\mathrm{a})}$ & $527.8 \pm 51.6^{\mathrm{a})}$ \\
\hline Body weight $(\mathrm{g})$ &
\end{tabular}

Animals were grouped according to the genotype of the D1Mit7 marker closest to the maximum lod scores for fat staining-positive area and fat staining-positive area/body weight within the chromosome 1 QTL region. Phenotypes are given as mean \pm SD. Means labeled with different superscripts are significantly different from one another $(P<0.05)$ by a one-way analysis of variance with Scheffe's $F$ analysis as post hoc test.

different genetic controls of fat storage in different fat tissues $[27,32]$.

We estimated the proportion of genetic variance and the effective number of genes for intramuscular fat content in the $F_{2}$ progeny. For the fat staining-positive area expressed as an absolute value, the proportion of genetic variance in the $\mathrm{F}_{2}$ was found to be about $69 \%$ by comparison of the variances in $F_{1}$ and $F_{2}$ progenies, and the number of genes was estimated roughly as 1 by Wright's formula [33]. Roughly $86 \%$ of the $\mathrm{F}_{2}$ variance in the fat staining-positive area as a percentage of the body weight appeared to be genetic, and the number of genes was estimated to be roughly 1 . These were consistent with the results of high heritability [18] and of presence of a major gene [16] for bovine marbling. Thus, detection of a significant QTL with large effect was expected in our experimental design, because it has been reported that there is a reasonable chance of detecting a QTL that explains $10 \%$ of phenotypic variance as a significant locus (lod score $\geq 4.3$ ), in an F2 intercross of 100 individuals [26]. However, indeed, only one suggestive QTL (4.3 > lod 
score $\geq 2.8$ ) was detected on chromosome 1. Further, this QTL was a small effect QTL that explains $5 \%$ and $7 \%$ of the total phenotypic variance and $7 \%$ and $8 \%$ of genetic variance for the fat staining-positive area as an absolute value and as a percentage of the body weight in the $\mathrm{F}_{2}$ progeny, respectively. The effective number of genes is calculated, assuming that the responsible genes are inherited with additive mode, have additive equal effects on the traits, and are not linked, and a failure of the assumption results in underestimation of the gene number [33]. Our result of detection of only one suggestive QTL with small effect suggests a failure of the assumption and the underestimation. Intraallelic interaction (heterosis), inter-allelic interaction (epistasis), genes with different effect sizes, and linked genes, respectively, which do not conform to the assumption of the additive mode of inheritance of the responsible genes, of the additive contributions of responsible genes to the traits, of the equal contributions of responsible genes to the traits, and of no linkage between responsible genes, may be involved in intramuscular fat accumulation in the OLETF. This phenotype in the OLETF might have a considerably higher degree of genetic complexity. Therefore, $-90 \%$ of genetic variance, which remain unexplained for the levels of intramuscular fat content, could be attributable to considerably complex genetic factors.

We have mapped the chromosome 1 QTL to $31 \mathrm{cM}$ 1-lod support interval. The human and mouse genome regions homologous to the interval of the QTL, respectively, would be $9 \mathrm{q} 12$ and $10 \mathrm{q} 23-\mathrm{q} 25.3$, and 19 (21-49 cM) [28]. Physiologically relevant candidate gene suggested by the homologous human and mouse regions is adrenergic alpha-2Areceptor (ADRA2A) locus [21]. The ADRA2A locus has been shown to associate with trunk to extremity skinfolds ratio [21]. In addition, Pfat3 locus [24], DAHL3 locus [10], and Dmol locus [29] may be defining the same underlying gene as the present QTL. The Pfat3, which has been previously identified as obesity QTL in mouse, affects adiposity in a cross between M16i and CAST/Ei [24]. The rat obesity QTLs, DAHL3 [10] and Dmol [29], have been reported to have an effect on body weight.

Recently, suggestive QTLs were identified for marbling on chromosomes 17 and 27 in cattle [3]. The interval of the chromosome 1 QTL detected in this study would correspond to the bovine genome regions, $8 \mathrm{q} 12-\mathrm{q} 18$ and 26q12-q23, but not chromosomes 17 and 27 [19]. Thus, the bovine genome regions of 8q12-q18 and 26q12-q23, in addition to chromosomes 17 and 27 , may be an attractive component for beef cattle breeding. In pig, it has been reported that a major gene influences intramuscular fat accumulation [8], and that QTLs for intramuscular fat content are located on porcine chromosomes 2, 4, 6 and 7 [4]. However, comparative map [19] suggests that these QTLs in pig would not be homologous to the chromosome 1 QTL detected in this study. Altogether, the data of the present and previous QTL studies [3, 4] are consistent with genetic heterogeneity of intramuscular fat accumulation.
ACKNOWLEDGEMENTS. We are grateful to Dr. Kazuya Kawano for kindly providing OLETF rats. This work was supported in part by research grant from the Ministry of Education, Science, Sports, and Culture of Japan.

\section{REFERENCES}

1. Bihoreau, M. T., Gauguier, D., Kato, N., Hyne, G., Lindpaintner, K., Rapp, J. P., James, M. R. and Lathrop, G. M. 1997. A linkage map of the rat genome derived from three $\mathrm{F}_{2}$ crosses. Genome Res. 7: 434-440.

2. Cameron, P. J., Zembayashi, M., Lunt, D. K., Mitsuhashi, T., Mitsumoto, T., Ozawa, S. and Smith, S. B. 1994. Relationship between Japanese beef marbling standard and intramuscular lipid in the M. longissimus thoracis of Japanese Black and American Wagyu cattle. Meat Sci. 38: 361-364.

3. Casas, E., Shackelford, S. D., Keele, J. W., Stone, R. T., Kappes, S. M. and Koohmaraie, M. 2000. Quantitative trait loci affecting growth and carcass composition of cattle segregating alternate forms of myostatin. J. Anim. Sci. 78: 560-569.

4. De Koning, D. J., Janss, L. L. G., Rattink, A. P., Van Oers, P. A. M., De Vries, B. J., Groenen, M. A. M., Van Der Poel, J. J., De Groot, P. N., (Pim) Brescamp, E. W. and Van Arendonk, J. A. M. 1999. Detection of quantitative trait loci for backfat thickness and intramuscular fat content in pigs (Sus scrofa). Genetics 152: 1679-1690.

5. Jacob, H. J., Brown, D. M., Bunker, R. K., Daly, M. J., Dzau, V. J., Goodman, A., Koike, G., Kren, V., Kurtz, T., Lernmark, Å., Levan, G., Mao, Y., Pettersson, A., Pravenec, M., Simon, J. S., Szpirer, C., Szpirer, J., Trolliet, M. R., Winer, E, S. and Lander, E. S. 1995. A genetic linkage map of the laboratory rat, Rattus norvegicus. Nat. Genet. 9: 63-69.

6. Jansen, R. C. 1993. Interval mapping of multiple quantitative trait loci. Genetics 135: 205-211.

7. Jansen, R. C. and Stam, P. 1994. High resolution of quantitative traits into multiple loci via interval mapping. Genetics $\mathbf{1 3 6}$ : 1447-1455.

8. Janss, L. L. G., Van Arendonk, J. A. M. and Brescamp, E. W. 1997. Bayesian statistical analyses for presence of single genes affecting meat quality traits in a crossed pig population. Genetics 145: 395-408.

9. Kanemoto, N., Hishigaki, H., Miyakita, A., Oga, K., Okuno, S., Tsuji, A., Takagi, T., Takahashi, E., Nakamura, Y. and Watanabe, T. K. 1998. Genetic dissection of "OLETF", a rat model for non-insulin-dependent diabetes mellitus. Mamm. Genome 9: 419-425.

10. Kato, N., Hyne, G., Bihoreau, M. T., Gauguier, D., Lathrop, G. M. and Rapp, J. P. 1999. Complete genome searches for quantitative trait loci controlling blood pressure and related traits in four segregating populations derived from Dahl hypertensive rats. Mamm. Genome 10: 259-265.

11. Kawano, K., Hirashima, T., Mori, S., Saitoh, Y., Kurosumi, M. and Natori, T. 1992. Spontaneous long-term hyperglycemic rat with diabetic complications Otsuka Long-Evans Tokushima Fatty (OLETF) strain. Diabetes 41: 1422-1428.

12. Lander, E. S. and Botstein, D. 1989. Mapping mendelian factors underlying quantitative traits using RFLP linkage maps. Genetics 121: 185-199.

13. Lander, E. and Kruglyak, L. 1995. Genetic dissection of complex traits: guidelines for interpreting and reporting linkage results. Nat. Genet. 11: 241-247.

14. Lander, E. S., Green, P., Abrahamson, J., Barlow, A., Daley, 
M., Lincoln, S. and Newburg, L. 1987. MAPMAKER: An interactive computer package for constructing primary genetic linkage maps of experimental and natural populations. Genomics 1: 174-181.

15. Lincoln, S. E. and Lander, E. S. 1992. Systematic detection of errors in genetic linkage data. Genomics 14: 604-610.

16. Miyake, T., Moriya, K. and Sasaki, Y. 1999. Influence of initial values on estimates by mixed inheritance model with Gibbs sampling. J. Anim. Breed. Genet. 116: 39-46.

17. Moralejo, D. H., Wei, S., Wei, K., Weksler-Zangen, S., Koike, G., Jacob, H. J., Hirashima, T., Kawano, K., Sugiura, K., Sasaki, Y., Ogino, T., Yamada, T. and Matsumoto, K. 1998. Identification of quantitative trait loci for non-insulin-dependent diabetes mellitus that interact with body weight in the Otsuka Long-Evans Tokushima Fatty rat. Proc. Assoc. Am. Physicians 110: 545-558.

18. Moriya, K., Dohgo, T. and Sasaki, Y. 1994. Restricted maximum likelihood estimation of heritabilities for carcass traits in the base and current populations of Japanese Black Cattle. Anim. Sci. Technol. 65: 720-725.

19. O'Brien, S. J., Menotti-Raymond, M., Murphy, W. J., Nash, W. G., Wienberg, J., Stanyon, R., Copeland, N. G., Jenkins, N. A., Womack, J. E. and Marshall Graves, J. A. M. 1999. The promise of comparative genomics in mammals. Science $\mathbf{2 8 6}$ : $458-462$.

20. Ogino, T., Wei, S., Wei, K., Moralejo, D. H., Kose, H., Mizuno, A., Shima, K., Sasaki, Y., Yamada, T. and Matsumoto, K. 2000. Genetic evidence for obesity loci involved in the regulation of body fat distribution in obese type 2 diabetes rat, OLETF. Genomics 70: 19-25.

21. Oppert, J. M., Tourville, J., Chagnon, M., Mauriege, P., Dionne, F. T., Perusse, L. and Bouchard, C. 1995. DNA polymorphisms in the $\alpha 2$ - and $\beta 2$-adrenoceptor genes and regional fat distribution in humans: association and linkage studies. Obesity Res. 3: 249-255.

22. Paterson, A. H., Lander, E. S., Hewitt, J. D., Peterson, S., Lincoln, S. E. and Tanksley, S. D. 1988. Resolution of quantitative traits into mendelian factors by using a complete linkage map of restriction fragment length polymorphisms. Nature (Lond.) 335: 721-726.

23. Pérusse, L., Chagnon, Y. C., Weisnagel, J. and Bouchard, C. 1999. The human obesity gene map: The 1998 update. Obesity Res. 7: 111-129.

24. Pomp, D. 1997. Genetic dissection of obesity in polygenic animal models. Behav. Genet. 27: 285-306.

25. Sugiura, K., Miyake, T., Taniguchi, Y., Yamada, T., Marolejo, D. H., Wei, S., Wei, K., Sasaki, Y. and Matsumoto, K. 1999.
Identification of novel non-insulin-dependent diabetes mellitus susceptibility loci in the Otsuka Long-Evans Tokushima Fatty rat by MQM-mapping method. Mamm. Genome 10: 11261131.

26. van Ooijen, J. W. 1992. Accuracy of mapping quantitative trait loci in autogamous species. Theor. Appl. Genet. 84: 803-811.

27. Warden, C. H., Fisler, J. S., Shoemaker, S. M., Wen, P-Z., Svenson, K. L., Pace, M. J. and Lusis, A. J. 1995. Identification of four chromosome loci determining obesity in a multifactorial mouse model. J. Clin. Invest. 95: 1545-1552.

28. Watanabe, T. K., Bihoreau, M-T., McCarthy, L. C., Kiguwa, S. L., Hishigaki, H., Tsuji, A., Browne, J., Yamasaki, Y., Mizoguchi-Miyakita, A., Oga, K., Ono, T., Okuno, S., Kanemoto, N., Takahashi, E., Tomita, K., Hayashi, H., Adachi, M., Webber, C., Davis, M., Kiel, S., Knights, C., Smith, A., Critcher, R., Miller, J., Thangarajah, T., Day, P. J. R., Hudson, J. R. Jr., Irie, Y., Takagi, T., Nakamura, Y., Goodfellow, P. N., Lathrop, M. G., Tanigami, A. and James, M. R. 1999. A radiation hybrid map of the rat genome containing 5,255 markers. Nat. Genet. 22: $27-36$

29. Watanabe, T. K., Okuno, S., Oga, K., Mizoguchi-Miyakita, A., Tsuji, A., Yamasaki, Y., Hishigaki, H., Kanemoto, N., Takagi, T., Takahashi, E., Irie, Y., Nakamura, Y. and Tanigami, A. 1999. Genetic dissection of "OLETF", a rat model for noninsulin-dependent diabetes mellitus: Quantitative trait locus analysis of (OLETF X BN) X OLETF. Genomics 53: 233-239.

30. Wei, S., Wei, K., Moralejo, D. H., Ogino, T., Koike, G., Jacob, H. J., Sugiura, K., Sasaki, Y., Yamada, T. and Matsumoto, K. 1999. Mapping and characterization of quantitative trait loci for non-insulin-dependent diabetes mellitus with an improved genetic map in the Otsuka Long-Evans Tokushima Fatty rat. Mamm. Genome 10: 249-258.

31. Wei, S., Wei, K., Moralejo, D. H., Yamada, T., Izumi, K. and Matsumoto, K. 1998. An integrated genetic map of the rat with 562 markers from different sources. Mamm. Genome 9: 10021007.

32. West, D. B., Goudey-Lefevre, J., York, B. and Truett, G. E. 1994. Dietary obesity linked to genetic loci on chromosomes 9 and 15 in a polygenic mouse model. J. Clin. Invest. 94: 14101416.

33. Wright, S. 1968. The genetics of quantitative variability. pp. 373-420. In: Evolution and the Genetics of Populations (Genetics and Biometrics Foundations ed.), University of Chicago Press, Chicago.

34. Zembayashi, M., Nabeta, H. and Mototsuji, T. 1988. Effects of breeds and nutritional planes on intramuscular lipid deposition of fattening of steers. Jpn. J. Zootech. Sci. 59: 39-48. 